•研究报告・

\title{
金钟藤入侵群落的种间联结及生态位特征
}

\author{
江 焕 ${ }^{1}$ 张 辉 ${ }^{1}$ 龙文兴 $^{1^{*}}$ 方燕山 ${ }^{2}$ 符明期 $^{2}$ 朱孔新 $^{3}$ \\ 1 (海南大学林学院, 海口 570228) \\ 2 (海南省黎母山省级自然保护区管理局, 海南琼中 572900) \\ 3 (海南省霸王岭林业局, 海南昌江 572700)
}

摘要: 金钟藤(Decalobanthus boisianus)是林业有害植物，其暴发生长和扩散对森林生态系统造成了严重破坏。本 文以海南岛 48 个金钟藤典型分布群落为研究对象, 用方差比率法和贡献定律法探究群落的稳定性; 用 $\chi^{2}$ 统计量、联 结系数(AC)、共同出现百分率(PC)、Ochiai指数和Dice指数分析金钟藤与伴生物种的种间联结关系; 用生态位宽度、 生态位相似性系数和生态位重叠指数研究群落中各物种的生态位特征, 以期为金钟藤生物防治的植物物种笁选提 供借鉴。结果表明: (1)金钟藤所在48个群落共有156种伴生植物, 其中大戟科、茜草科、桑科、无患子科和樟科植 物占优势; (2)群落中优势物种呈正联结关系, 植物种类累积倒数百分比与累积相对频度交点坐标为(44.53, 55.47), 远离稳定交点坐标(20,80), 说明群落处于不稳定状态; (3)金钟藤与芳槁润楠(Machilus suaveolens)、黄椿木姜子 (Litsea variabilis)、岭南山竹子(Garcinia oblongifolia)、显脉杜英(Elaeocarpus dubius)、鸭脚木(Schefflera octophylla) 和银柴(Aporusa dioica)都紧密关联, 说明金钟藤与这些物种的资源利用方式较相似; (4)金钟藤的生态位宽度最大, 与伴生物种间的生态位重叠度较高, 但伴生物种间的生态位重叠度较低。金钟藤的入侵导致群落处于不稳定状态, 并与伴生物种间存在激烈的竞争关系。因此，建议在金钟藤已入侵的群落中大量栽种芳槁润楠、黄椿木姜子、显 脉杜英、鸭脚木和银柴, 以遏制其蔓延; 大量栽种翻白叶树 (Pterospermum heterophyllum)、海南菜豆树 (Radermachera hainanensis)、九节(Psychotria rubra) 和肉实树(Sarcosperma laurinum) 用于金钟藤入侵群落的植被恢复。 关键词: 金钟藤; 伴生物种; 群落稳定性; 种间联结; 生态位

\section{Interspecific associations and niche characteristics of communities invaded by Decalobanthus boisianus}

\author{
Huan Jiang ${ }^{1}$, Hui Zhang ${ }^{1}$, Wenxing Long ${ }^{1 *}$, Yanshan Fang ${ }^{2}$, Mingqi Fu ${ }^{2}$, Kongxin Zhu ${ }^{3}$ \\ 1 College of Forestry, Hainan University, Haikou 570228 \\ 2 Administration of Limushan Natural Reserve, Qiongzhong, Hainan 572900 \\ 3 Forestry Bureau of Bawangling, Changjiang, Hainan 572700
}

\begin{abstract}
Decalobanthus boisianus is a noxious weed that has seriously damaged tropical forest ecosystems on Hainan Island due to its rapid dispersal and growth abilities. We investigated 48 communities invaded by D. boisianus and explored the interspecific associations between the community species using $\chi^{2}$ tests, association coefficient (AC), percentage of co-occurrence (PC), and the Ochiai and Dice indices. We assessed community stability using the variance ratio $(V R)$ and contribution law method, and examined niche width, niche similarity and niche overlap of $D$. boisianus and companion species. Results showed that there were 156 companion species in the D. boisianus communities, belonging to Euphorbiaceae, Rubiaceae, Moraceae, Sapindaceae and Lauraceae. There were positive associations between dominant species. The coordinate (44.53, 55.47) between the inverse accumulative percentage of species and their accumulative relative frequency was far from the stability point $(20,80)$. This indicates that these communities are in an unstable state. Machilus suaveolens, Litsea variabilis, Garcinia oblongifolia, Elaeocarpus dubius, Schefflera octophylla and Aporusa dioica were closely associated with D. boisianus, indicating that these species have
\end{abstract}


similar resource requirements. Decalobanthus boisianus had the highest niche width which overlapped greatly with companion species. The niches among companion species seldomly overlapped. Our results suggest that the invasion of $D$. boisianus led to an unstable community with intense competition between companion species and D. boisianus. Our findings can be helpful in species selection for biologically controlling to $D$. boisianus in tropical areas.

Key words: Decalobanthus boisianus; companion species; community stability; interspecific association; niche

生物入侵不仅对入侵生态系统的完整性与稳 定性产生巨大的破坏(Finch, 2012), 同时也会威胁 生态系统的结构和功能, 被列为导致全球生物多样 性丧失的第二大因素(Pimentel, 2000; Callaway \& Maron, 2006)。在研究生物入侵的危害和机制时, 除 入侵生物自身的特征外, 其对入侵群落的影响及群 落中其他物种的响应也是研究重点(郭连金和王涛, 2009; 刘海等, 2017), 往往以各物种间的联结关系、 生态位特征以及群落的稳定性研究作为切入点(洪 思思等, 2008; 郭连金和王涛, 2009; 陈䚲等, 2012; 刘海等, 2017)。

种间联结是指不同物种在空间分布上的相互 关联性(Gleason, 1926), 包括空间分布和物种之间 的功能依赖关系(彭少麟等, 1999; 陈玉凯等, 2011), 展现了种间竞争的结果或群落的现状(徐满厚等, 2016), 是物种之间相互依存、竞争或随机出现等情 况的综合反映(Hubalek, 2008)。生态位是物种对多 个环境因子生态适应的综合结果, 反映了物种在群 落中的时间、空间位置以及功能、地位(Hutchinson, 1957)。群落稳定性是植物群落结构与功能的综合特 征, 是种群自身调节、种间竞争及联结的集中反映 (高润梅等, 2012; 刘海等, 2017)。生态位与种间联 结关系共同解释了群落中物种的空间分布及其对 资源的利用特征, 两者均会影响群落稳定性(史作 民等, 1999; 李帅锋等, 2011)。因此, 将群落稳定性、 种间联结关系以及生态位理论结合, 能够全面有效 地反映群落的基本结构及发展状态, 有助于了解优 势物种对资源利用的生态习性及其相互关系, 对生 态系统的经营管理和植被恢复等具有指导意义 (Rosenthal, 2003; Thevathasan \& Gordon, 2004)。

金钟藤(Decalobanthus boisianus)是旋花科鱼黄 草属多年生常绿藤本植物, 既能生长于潮湿的土壤, 也能忍耐一定的干旱(李玲等, 2008); 既可通过种 子繁殖, 又具有很强的无性繁殖能力, 能快速入侵
到郁闭度较低的人工林、灌木林和次生林, 并迅速 攀爬至植物顶端, 导致底层植物无法接收充足的阳 光而死亡(王伯荪等, 2005; 吴林芳等, 2007; 黄乔乔 等, 2013), 使森林生态系统崩溃, 被国家林业局列 为重大林业有害生物(梁玮莎等, 2012)。海南岛是金 钟藤的主要分布区域之一(王伯称等, 2005)。自20世 纪90年代以来, 金钟藤在海南岛呈不断蔓延扩散的 趋势, 入侵到中西部的各个县市, 在某些林区, 其 冠层的连片面积可高达10 ha (王伯荪等, 2009)。研 究者对金钟藤的分类学考证(王伯荪等, 2007)、地理 分布(王伯荪等, 2005, 2009)、生理特征(李玲等, 2008; Huang et al, 2013; 李晓霞等, 2014)以及防治 应用(童国建等, 2005; 吴林芳等, 2007)等均作过详 细的探讨。但是, 金钟藤的入侵会给群落带来怎样 的影响? 与群落伴生物种存在怎样的联结关系? 群 落中各个物种又具有怎样的生态位特征? 这些都 有待揭示。本文以海南岛的 48 个金钟藤典型群落为 研究对象, 拟对群落的稳定性、优势树种的种间联 结以及生态位特征开展研究。我们假设: (1)金钟藤 对资源的抢夺会导致群落处于不稳定状态; (2)群落 中的部分优势种与金钟藤存在竞争关系, 表现为显 著的联结关系与生态位重叠。

\section{材料与方法}

\section{1 研究区域概况}

海南岛位于中国最南端, 总面积为 3.54 万 $\mathrm{km}^{2}$, 地理坐标为 $18^{\circ} 10^{\prime}-20^{\circ} 10^{\prime} \mathrm{N}, 108^{\circ} 37^{\prime}-111^{\circ} 03^{\prime} \mathrm{E}$ 。海 南岛整体为中间高四周低, 四周主要由山地丘陵和 平原组成。岛上主要为热带海洋性季风气候, 年均 温度22.5-25.6 ${ }^{\circ} \mathrm{C}$, 最低温和最高温分别出现在1月 和7月, 年平均降水量923-2,459 mm, 有明显的雨 季和干季, 5-10月为雨季, 最大降水量出现在8、9 月。降雨空间分布为东部多于西部(胡小婵和高宏华, 2008)。 


\section{2 样方选取及野外调查}

2017年10-12月，在海南岛西南部的昌江和白 沙, 西部的乐东, 中部的琼中、五指山和琼海, 中北 部的陵水以及西北部的保亭和三亚 9 个县市, 选择 金钟藤典型分布的区域，设置 48 个面积为 $20 \mathrm{~m} \times 20$ $\mathrm{m}$ 的样方, 调查样方内出现的所有植物种类、胸径、 高度、多度、频度等。剔除偶见种后，计算样地内 所有物种的相对重要值, 用于表示物种在群落中的 优势程度，取相对重要值大于 1.0 且频度大于 15 的 优势种进行数据分析(表1)。相对重要值计算公式 如下:

相对重要值 $=($ 相对频度 + 相对密度 + 相对显著度 $) / 3$

\section{3 数据分析}

\subsection{1 总体联结性检验}

采用Schluter (1984)提出的方差比率法测定金 钟藤群落的总体关联性, 再通过统计量 $W$ 检验关联 显著性。计算公式如下：

$$
\begin{aligned}
& \delta \text { 争 }=\sum_{i=1}^{S} P_{i}\left(1-{ }_{i}\right) \\
& S_{T}^{2}=(1 / N) \sum_{j=1}^{N}\left(T_{j}-t\right)
\end{aligned}
$$

$$
\begin{aligned}
& V R=S_{T}^{2} / \delta_{\mathrm{T}}^{2} \\
& P_{i}=n_{i} / N
\end{aligned}
$$

式中, $S$ 为总物种数, $N$ 为总样方数, $T_{j}$ 为样方 $j$ 内出现 的物种数, $n_{i}$ 为物种 $i$ 出现的样方数, $t$ 为样方中种的 平均数。

$V R>1$ 表示物种间总体上表现为正关联, $<1$ 则 表示负关联。由于种间的正负关联可以相互抵消, 因此需采用统计量 $W$ 进一步检验 $V R$ 值相对 1 的偏移 程度, 如果 $\chi_{0.95}^{2}(N)<W<\chi_{0.05}^{2}(N)$, 表示物种间无 显著关联性，反之表示物种间显著相关联。

\subsection{2 种间联结关系的检验}

种间联结关系采用 $\chi^{2}$ 统计量、联结系数(AC)、 共同出现百分率(PC)、Ochiai指数和Dice指数等方 法计算。通过建立种对间的 $2 \times 2$ 列联表，统计出 $a$ 、 $b 、 c 、 d$ 值。其中, $a$ 为 $\mathrm{A} 、 \mathrm{~B}$ 两物种同时存在的样方 数, $b$ 为仅 $\mathrm{B}$ 物种存在而 $\mathrm{A}$ 物种不存在的样方数, $c$ 为 $\mathrm{A}$ 物种存在而 $\mathrm{B}$ 物种不存在的样方数, $d$ 为两物种均 不存在的样方数。为避免分母为 0 而出现无法计算 的情况，将 $b$ 和 $d$ 值加权为 1 , 以获得较为客观的效 果(王伯荪和彭少麟, 1985)。

(1) $\chi^{2}$ 统计量

由于取样的非连续性, $\chi^{2}$ 值采用Yates的连续校

\begin{tabular}{|c|c|c|c|c|c|}
\hline $\begin{array}{l}\text { 序号 } \\
\text { No. }\end{array}$ & $\begin{array}{l}\text { 种名 } \\
\text { Species }\end{array}$ & $\begin{array}{l}\text { 相对频度 } \\
\text { Relative frequency (\%) }\end{array}$ & $\begin{array}{l}\text { 相对密度 } \\
\text { Relative density (\%) }\end{array}$ & $\begin{array}{l}\text { 相对显著度 } \\
\text { Relative prominence (\%) }\end{array}$ & $\begin{array}{l}\text { 重要值 } \\
\text { Importance value }\end{array}$ \\
\hline 1 & 金钟藤 Decalobanthus boisianus & 4.53 & 14.88 & 2.21 & 7.21 \\
\hline 2 & 鸭脚木 Schefflera octophylla & 1.89 & 4.27 & 3.89 & 3.35 \\
\hline 3 & 水锦树 Wendlandia uvariifolia & 1.42 & 2.22 & 1.32 & 1.65 \\
\hline 4 & 黄椿木姜子 Litsea variabilis & 1.89 & 2.09 & 0.72 & 1.57 \\
\hline 6 & 假柿木姜子 Litsea monopetala & 1.13 & 1.41 & 1.63 & 1.39 \\
\hline 7 & 倒吊笔 Wrightia pubescens & 1.32 & 1.58 & 1.24 & 1.38 \\
\hline 8 & 芳槁润楠 Machilus suaveolens & 1.61 & 1.21 & 1.28 & 1.37 \\
\hline 9 & 翻白叶树 Pterospermum heterophyllum & 0.94 & 1.31 & 1.76 & 1.34 \\
\hline 10 & 肉实树 Sarcosperma laurinum & 1.32 & 1.25 & 1.11 & 1.23 \\
\hline 12 & 楝叶吴茱实 Evodia glabrifolia & 1.04 & 0.64 & 1.87 & 1.18 \\
\hline 13 & 九节 Psychotria rubra & 1.23 & 2.05 & 0.13 & 1.14 \\
\hline 14 & 银柴 Aporusa dioica & 1.61 & 1.31 & 0.43 & 1.11 \\
\hline 15 & 显脉杜英 Elaeocarpus dubius & 1.42 & 0.71 & 1.1 & 1.07 \\
\hline 16 & 海南菜豆树 Radermachera hainanensis & 1.13 & 0.98 & 1.04 & 1.05 \\
\hline 17 & 白楸 Mallotus paniculatus & 1.23 & 0.81 & 1.08 & 1.04 \\
\hline
\end{tabular}

表1 金钟藤群落优势种的相对频度、相对密度、相对显著度及重要值

Table 1 Relative frequency, relative density, relative prominence and importance value of dominant plant species in Decalobanthus boisianus community 
正公式(Greig-Smith, 1983)计算:

$$
\chi^{2}=\frac{N\left[(|a d-b c|)-\frac{1}{2} N\right]^{2}}{(a+b)(c+d)(a+c)(b+d)}
$$

式中, $N$ 为总样方数。当 $a d>b c$ 时, 种对间呈正联结; 当 $a d<b c$ 时, 种对间呈负联结。 $\chi^{2}<3.841$ 时, 表示 种对间联结不显著; 当 $3.841<\chi^{2}<6.635$ 时, 表示种 对间存在一定的生态联结; 当 $\chi^{2}>6.635$ 时, 表示种 对间存在显著生态联结。

$\chi^{2}$ 统计量能较客观、精确地反映种对间联结的 显著程度, 但不能量化说明物种联结的紧密程度, 在一定程度上模糊了种间关联性之间的差异性。因 此, 需结合其他系数作进一步分析。

\section{(2)联结系数}

联结系数(AC)虽可进一步体现种间的联结程 度, 但容易夸大两物种均不出现时种对间的联结显 著程度(王伯䔉和彭少麟, 1985)。其计算公式如下:

当 $a d \geq b c$ 时, $\mathrm{AC}=(a d-b c) /[(a+b)(b+d)]$;

当 $b c>a d$ 且 $d \geq a$ 时,

$\mathrm{AC}=(a d-b c) /[(a+b)(a+c)] ;$

当 $b c>a d$ 且 $d<a$ 时,

$\mathrm{AC}=(a d-b c) /[(b+d)(d+c)]$

$\mathrm{AC}$ 值域为 $[-1,1]$, 越接近 1 , 说明物种间的正 联结性越强; 越接近 -1 , 说明负联结性越强; $\mathrm{AC}=$ 0 说明物种间完全独立。

\section{(3)共同出现百分率}

共同出现百分率(PC)可体现物种间正联结程度 的强弱, 但容易夸大 $a 、 b$ 和 $c$ 值在联结性测定中的作 用。其计算公式为 $\mathrm{PC}=a /(a+b+c)$ 。 PC值域为 $[0,1]$, 越接近 1 , 表明正联结程度越高, 两物种共同出现 或共同不出现的可能性越大, 两物种的生态习性和 对环境的需求越一致(Cárdenas et al, 2014)。

(4) Ochiai指数和Dice指数

Ochiai指数 (OI) 和Dice指数(DI) 能较精确地反 映不同种对间相伴出现的机率和联结程度, 并克服 了点联结系数受 $d$ 值影响较大造成的偏差(Cárdenas et al, 2014)。计算公式如下:

$$
\begin{aligned}
& \mathrm{OI}=a / \sqrt{(a+b)(a+c)} \\
& \mathrm{DI}=2 a /(2 a+b+c)
\end{aligned}
$$

这两个指数的值域均为 $[0,1]$, 越接近 1 , 表明 种对间的正联结程度越高; 反之则越低。因此, 本 研究以 $\chi^{2}$ 检验为基础来定性测定物种间联结性的显 著性, 再结合AC、PC、OI和DI等指数进行综合分
析，能更科学准确地判定金钟藤群落的种间联结关 系。同时, 本研究中选取了重要值大于1且频度大于 15的物种进行种间关联分析, 也能较有效地避免由 上述指数的缺陷造成的影响。

\subsection{3 群落稳定性分析}

根据改良过的贡献定律法, 首先将群落的优势 物种按频度大小依次排列, 换算为相对频度后逐步 累积; 接着将样地内植物种类的总和取倒数, 按照 植物种类排列顺序也逐步对应累积; 然后以植物种 类倒数累计值为横坐标 $(x)$, 累积相对频度为纵坐标 (y), 建立散点平滑曲线模型; 最后在横纵坐标 100 处直线连接, 其与曲线交点即群落稳定比值。该比 值越接近(20, 80), 群落就越稳定(Godron, 1972; 郑 元润, 2000)。

平滑曲线模拟方程为:

$y=\mathrm{A} x^{2}+\mathrm{B} x+\mathrm{C}$

直线方程为:

$y=100-x$

将直线方程代入平滑曲线模拟方程:

$\mathrm{A} x^{2}+(\mathrm{B}+1) x+\mathrm{c}-100=0$

解得交点横坐标 $x$ 为:

$$
x=\frac{-(\mathrm{B}+1) \pm \sqrt{(\mathrm{B}+1)^{2}-4 \mathrm{~A}(\mathrm{C}-100)}}{2 \mathrm{~A}}
$$

再将该解代入式(13)求得交点纵坐标 $y$ 。

\subsection{4 金钟藤与伴生物种的生态位分析}

在本研究中, 将每个 $20 \mathrm{~m} \times 20 \mathrm{~m}$ 样方视为一个 综合资源位，并以 17 个优势种在各个资源位的相对 重要值作为指标, 由此得出的结果能较好地体现物 种对环境资源的利用效率，同时也能避免因各物种 的个体大小差异所带来的误差(张继义等, 2003; 陈 㶦等, 2012; 汤景明等, 2012)。

\section{(1)生态位宽度}

生态位宽度反映物种对资源的利用情况和物 种的分布与数量, 采用Shannon-Wiener指数公式计 算(Levins, 1968):

$$
\begin{aligned}
& B_{i}=-\sum_{j=1}^{r}\left(P_{i j} \ln P_{i j}\right) \\
& P_{i j}=n_{i j} / N_{i}
\end{aligned}
$$

式中, $B_{i}$ 为物种 $i$ 的生态位宽度, 值域为 $[0, \ln r], P_{i j}$ 为 种 $i$ 在资源 $j$ 上的重要值占该种在所有资源上的重要 值的比例, $r$ 为资源总位数即样方数; $n_{i j}$ 为物种 $i$ 在资 
源 $\mathrm{j}$ 的重要值, $N_{i}$ 表示物种 $\mathrm{i}$ 在所有资源上的重要值 之和。

\section{(2)生态位重叠}

生态位重叠反映了物种之间利用资源或对环 境适应能力的相似程度, 本研究采用以下 2 种方式 进行计算:

\section{(i)生态位相似性系数}

生态位相似性系数反映两个物种利用资源的 相似程度，其计算公式(Schoener, 1974)为:

$$
C_{i h}=1-0.5 \sum_{j=1}^{r}\left|P_{i j}-P_{h j}\right|
$$

式中, $C_{i h}$ 为物种 $i$ 与物种 $h$ 的相似性程度, 值域为 $[0$, 1]; $P_{i j}$ 和 $P_{h j}$ 分别为种 $i$ 和种 $h$ 在资源 $j$ 上的重要值百分 率, 且 $C_{i h}=C_{h i}$ 。

\section{(ii)生态位重叠指数}

生态位重叠指数反映两个物种利用同等级资 源且相互重叠的情况，其计算公式(Pianka, 1973)为:

$$
O_{i k}=\sum_{j=1}^{r} P_{i j} P_{k j} / \sqrt{\sum_{j=1}^{r} P_{i j}^{2} \sum_{j=1}^{r} P_{k j}^{2}}
$$

式中, $O_{i k}$ 为物种 $i$ 和物种 $k$ 的重叠指数; $P_{i j}$ 和 $P_{k j}$ 分别 为种 $i$ 和种 $k$ 在资源 $j$ 上的重要值。

所有的数据分析和制图均通过R 3.5.1的spaa包 实现。

\section{结果}

\section{1 主要种对间的关联性}

根据 $\chi^{2}$ 统计量(表2)和联结系数分析结果(图1), 在136个种对中, 共有69个种对表现出正关联。其中, 黄椿木姜子 (Litsea variabilis)-九节 (Psychotria rubra)、岭南山竹子(Evodia glabrifolia)-显脉杜英 (Elaeocarpus dubius) 表现出极显著正联结 $\left(\chi^{2}>\right.$ 6.635); 倒吊笔(Wrightia pubescens)-黄椿木姜子、岭 南山竹子-银柴(Aporusa dioica)、鸭脚木(Schefflera octophylla)-银柴表现为显著的正联结关系 $(3.841<$ $\left.\chi^{2}<6.635\right)$ 。倒吊笔-肉实树(Sarcosperma laurinum) 和翻白叶树 (Pterospermum heterophyllum)-岭南山 竹子表现为显著的负联结关系 $\left(\chi^{2}>6.635\right.$ 但AC < $-0.5)$ 。

根据共同出现百分比(图2a)、Ochiai指数(图2b) 和Dice指数(图2c)的分析结果，金钟藤-芳槁润楠 (Machilus suaveolens)、金钟藤-黄椿木姜子、金钟 藤-岭南山竹子、金钟藤-显脉杜英、金钟藤-鸭脚 木、金钟藤-银柴、岭南山竹子-显脉杜英、岭南山 竹子-银柴、显脉杜英-银柴和鸭脚木-显脉杜英表 现出非常紧密的关联性, 相伴出现的机率最大; 倒 吊笔一肉实树、九节-楝叶吴茱莫(Evodia glabrifolia)、翻白叶树-岭南山竹子、白楸(Mallotus

表2 金钟藤群落优势种间 $\chi^{2}$ 统计量检验

\begin{tabular}{|c|c|c|c|c|c|c|c|c|c|c|c|c|c|c|c|c|}
\hline 编号 No. & 1 & 2 & 3 & 4 & 5 & 6 & 7 & 8 & 9 & 10 & 11 & 12 & 13 & 14 & 15 & 16 \\
\hline 2 & 0.39 & & & & & & & & & & & & & & & \\
\hline 3 & -0.09 & 0.15 & & & & & & & & & & & & & & \\
\hline 4 & 0.26 & 0.83 & 0.33 & & & & & & & & & & & & & \\
\hline 5 & -0.26 & 0.06 & 0.00 & 0.03 & & & & & & & & & & & & \\
\hline 6 & -0.21 & 1.26 & 0.94 & 0.02 & 0.02 & & & & & & & & & & & \\
\hline 7 & -0.16 & 0.68 & 0.02 & 5.15 & 0.01 & 2.39 & & & & & & & & & & \\
\hline 8 & 0.32 & 1.29 & 0.03 & 1.54 & 0.01 & 2.46 & 0.22 & & & & & & & & & \\
\hline 9 & -0.09 & 2.02 & 0.11 & 3.19 & 0.00 & 0.75 & 1.19 & 0.03 & & & & & & & & \\
\hline 10 & -0.16 & 3.48 & 0.49 & 0.95 & 0.05 & 1.46 & 4.88 & 0.02 & 0.01 & & & & & & & \\
\hline 11 & 0.39 & 0.73 & 2.55 & 0.70 & 0.11 & 1.26 & 0.13 & 1.40 & 4.11 & 0.01 & & & & & & \\
\hline 12 & 0.00 & 0.04 & 0.01 & 0.44 & 0.44 & 0.62 & 0.99 & 0.15 & 0.01 & 0.32 & 0.67 & & & & & \\
\hline 13 & -0.21 & 1.26 & 0.13 & 6.29 & 0.02 & 0.01 & 0.90 & 0.61 & 0.75 & 0.00 & 0.40 & 1.22 & & & & \\
\hline 14 & 0.16 & 4.03 & 0.02 & 0.01 & 2.80 & 0.06 & 0.35 & 0.02 & 0.23 & 0.05 & 4.03 & 0.13 & 0.12 & & & \\
\hline 15 & 0.21 & 1.26 & 0.13 & 0.02 & 0.02 & 1.66 & 0.06 & 0.96 & 0.75 & 0.00 & 8.30 & 0.02 & 0.01 & 0.90 & & \\
\hline 16 & -0.16 & 0.68 & 0.02 & 0.23 & 1.69 & 0.06 & 1.43 & 0.22 & 2.92 & 0.05 & 0.13 & 0.08 & 2.39 & 0.35 & 0.06 & \\
\hline 17 & -0.12 & 0.45 & 0.01 & 0.14 & 0.68 & 0.37 & 0.05 & 0.20 & 0.49 & 0.59 & 0.27 & 0.01 & 1.46 & 0.98 & 0.00 & 0.15 \\
\hline
\end{tabular}

Table $2 \chi^{2}$ correlation test of dominant plant species in Decalobanthus boisianus community

物种编号含义见表1。Species codes are the same as in Table 1 . 


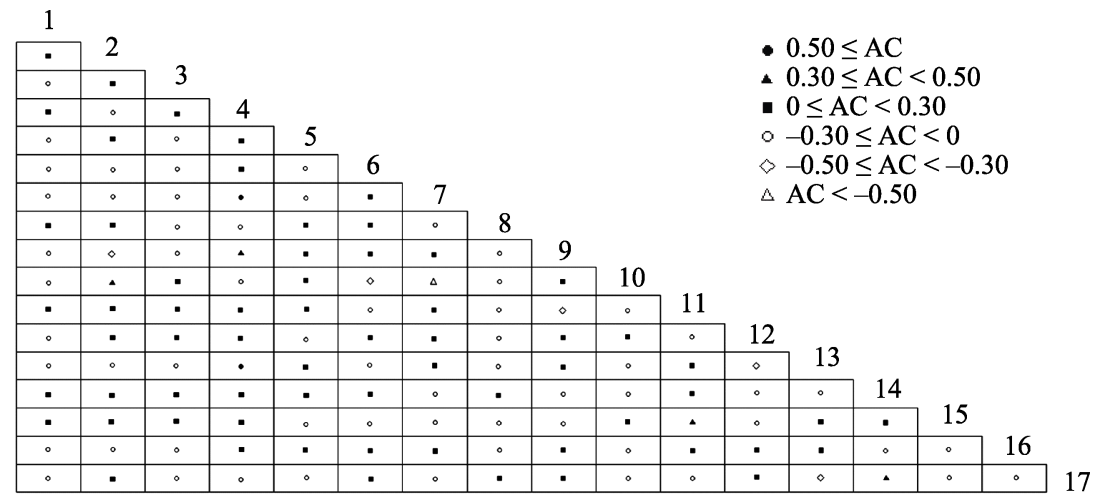

图1 金钟藤群落主要优势种种间联结系数(AC)半矩阵图。物种编号含义见表1。

Fig. 1 Semi-matrix diagram of association coefficients (AC) of dominant plant species in Decalobanthus boisianus community. Species codes are the same as in Table 1.
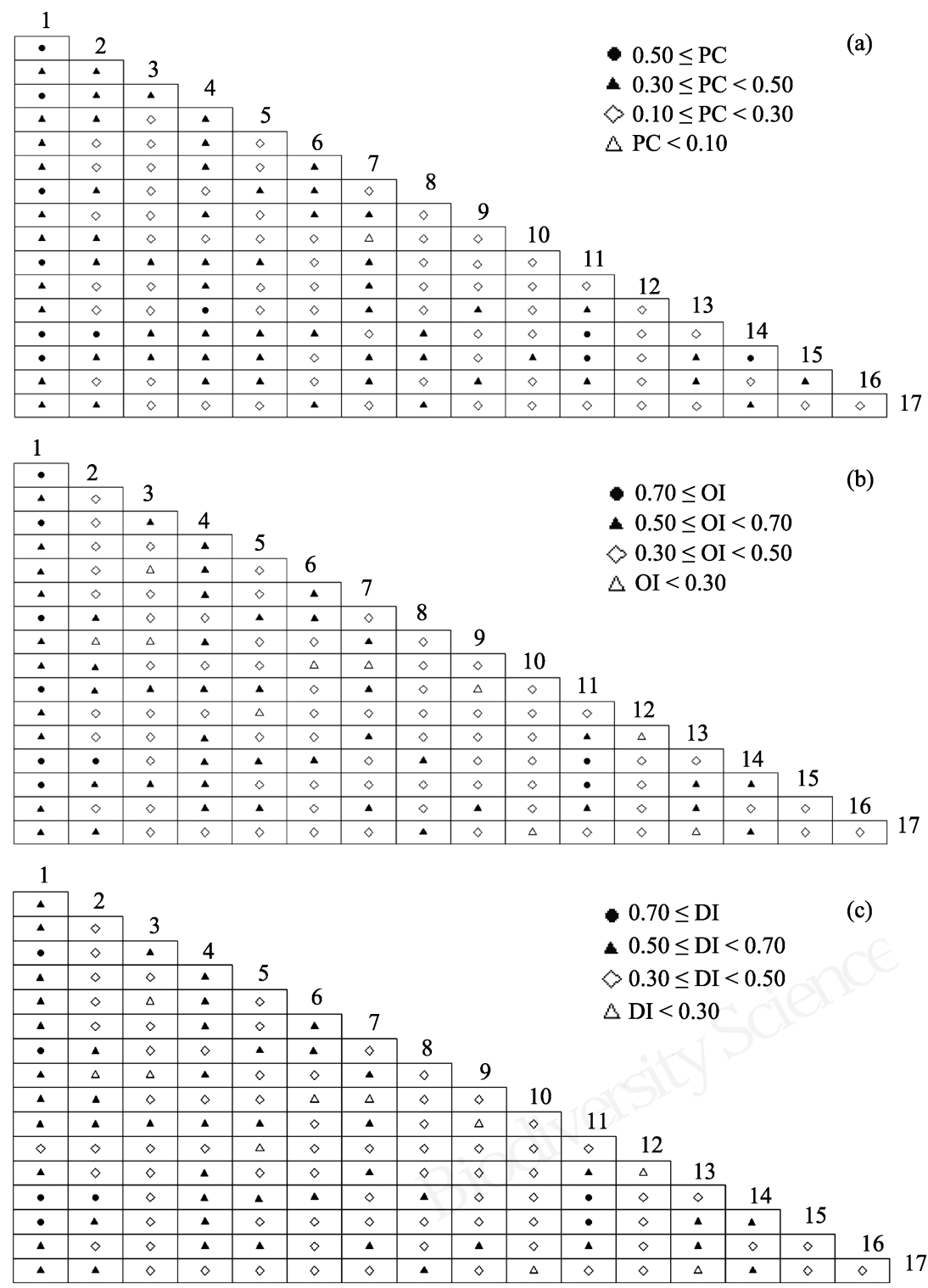

图2 金钟藤群落主要优势种共同出现百分率(PC)、Ochiai指数(OI)和Dice指数(DI)半矩阵图。物种编号含义见表1。

Fig. 2 Semi-matrix diagram of co-occurence rate (PC), Ochiai index (OI), and Dice index (DI) of dominant species in Decalobanthus boisianus community. Species codes are the same as in Table 1. 
paniculatus)-九节和假柿木姜子(Litsea monopetala)肉实树的关联性最弱。

\section{2 种群间的总体联结性与群落稳定性}

金钟藤所在 48 个群落共有 156 种伴生植物, 隶 属于53科91属。其中, 大戟科(8属20种)、茜草科(6 属6种)、桑科(3属12种)、无患子科(7属7种)、樟科 (6属10种)等种类较多。群落中主要优势物种间呈不 显著的正关联关系 $(V R=1.30, W=62.36$, CI (33.10, 65.17))。平滑曲线模拟方程与直线方程的交点为 (44.53, 55.47), 远离稳定交点坐标(20,80), 说明群 落处于不稳定状态(图3)。

\section{3 金钟藤与伴生物种的生态位分析}

17 个优势物种中, 金钟藤的生态位宽度最高 (3.83), 倒吊笔最低(2.47)。芳槁润楠(3.07)、黄椿木 姜子(3.04)、显脉杜英(3.09)和银柴(3.10)与金钟藤的 生态位宽度较为相近(表3)。

生态位相似性系数与生态位重叠指数在 0.5 以 上的有 19 对, 占总数的 $13.97 \%$ (表3), 且大多数发 生在金钟藤与伴生植物间, 说明这些种对在环境资 源不足时会发生激烈的竞争。多数种对的指数介于
0.1-0.5之间, 说明存在一定的竞争关系, 但在资源 丰富的时候仍能共存; 倒吊笔一肉实树、翻白叶树鸭脚木、假柿木姜子 - 肉实树和九节-水锦树 (Wendlandia uvariifolia)间的指数小于 0.1 , 说明这些 种对之间几乎不存在竞争关系。

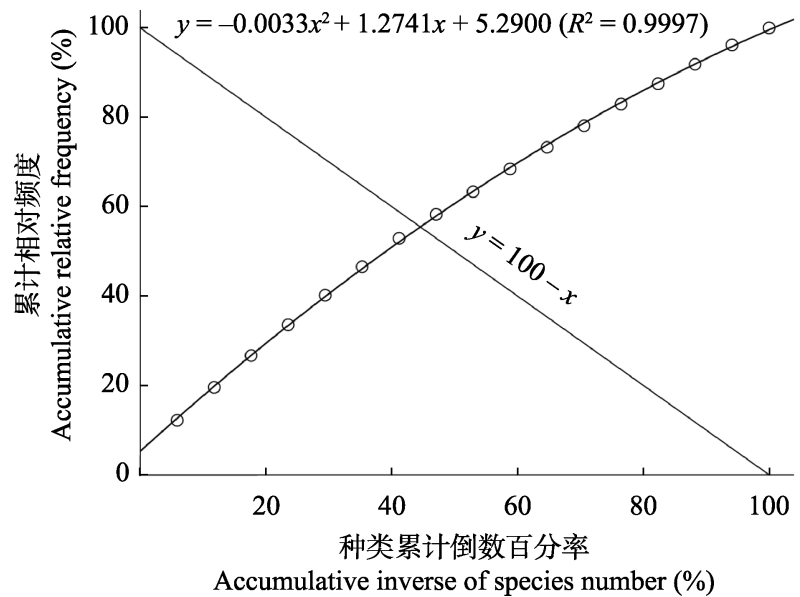

图3 金钟藤群落稳定性分析图

Fig. 3 The stability graph for Decalobanthus boisianus community

表3 金钟藤群落主要优势种间的生态位相似性比例(对角线上)和生态位重叠指数(对角线下)

Table 3 Niche similarity (above the diagonal) and niche overlap (below the diagonal) of dominant plant species in Decalobanthus boisianus community

\begin{tabular}{|c|c|c|c|c|c|c|c|c|c|c|c|c|c|c|c|c|c|c|}
\hline $\begin{array}{l}\text { 编号 } \\
\text { No. }\end{array}$ & $\begin{array}{l}\text { 生态位 } \\
\text { 宽度 } \\
\text { Niche } \\
\text { width }\end{array}$ & 1 & 2 & 3 & 4 & 5 & 6 & 7 & 8 & 9 & 10 & 11 & 12 & 13 & 14 & 15 & 16 & 17 \\
\hline 1 & 3.83 & - & 0.42 & 0.32 & 0.51 & 0.41 & 0.38 & 0.35 & 0.52 & 0.36 & 0.39 & 0.40 & 0.32 & 0.40 & 0.54 & 0.51 & 0.37 & 0.39 \\
\hline 2 & 2.82 & 0.54 & - & 0.27 & 0.30 & 0.21 & 0.18 & 0.15 & 0.52 & 0.09 & 0.41 & 0.27 & 0.33 & 0.17 & 0.42 & 0.28 & 0.19 & 0.31 \\
\hline 4 & 3.04 & 0.64 & 0.25 & 0.33 & - & 0.32 & 0.34 & 0.38 & 0.24 & 0.36 & 0.18 & 0.30 & 0.31 & 0.50 & 0.34 & 0.29 & 0.37 & 0.25 \\
\hline 5 & 2.61 & 0.45 & 0.13 & 0.17 & 0.29 & - & 0.23 & 0.19 & 0.24 & 0.22 & 0.23 & 0.21 & 0.14 & 0.25 & 0.42 & 0.26 & 0.31 & 0.15 \\
\hline 6 & 2.69 & 0.41 & 0.12 & 0.15 & 0.27 & 0.17 & - & 0.25 & 0.34 & 0.20 & 0.14 & 0.27 & 0.39 & 0.19 & 0.34 & 0.19 & 0.20 & 0.24 \\
\hline 7 & 2.47 & 0.35 & 0.21 & 0.15 & 0.42 & 0.24 & 0.11 & - & 0.22 & 0.23 & 0.09 & 0.24 & 0.29 & 0.26 & 0.22 & 0.30 & 0.38 & 0.26 \\
\hline 8 & 3.07 & 0.63 & 0.68 & 0.42 & 0.25 & 0.11 & 0.28 & 0.11 & - & 0.24 & 0.40 & 0.23 & 0.23 & 0.24 & 0.40 & 0.26 & 0.27 & 0.30 \\
\hline 10 & 2.68 & 0.54 & 0.56 & 0.26 & 0.14 & 0.16 & 0.09 & 0.04 & 0.58 & 0.20 & - & 0.23 & 0.33 & 0.17 & 0.24 & 0.34 & 0.22 & 0.14 \\
\hline 11 & 2.78 & 0.37 & 0.17 & 0.35 & 0.22 & 0.18 & 0.19 & 0.14 & 0.21 & 0.11 & 0.23 & - & 0.19 & 0.21 & 0.36 & 0.49 & 0.22 & 0.24 \\
\hline 12 & 2.57 & 0.46 & 0.45 & 0.39 & 0.31 & 0.11 & 0.54 & 0.27 & 0.30 & 0.30 & 0.31 & 0.13 & - & 0.12 & 0.29 & 0.26 & 0.19 & 0.23 \\
\hline 13 & 2.67 & 0.50 & 0.12 & 0.08 & 0.56 & 0.26 & 0.13 & 0.17 & 0.23 & 0.61 & 0.12 & 0.11 & 0.12 & - & 0.22 & 0.23 & 0.46 & 0.17 \\
\hline 14 & 3.10 & 0.62 & 0.50 & 0.30 & 0.37 & 0.39 & 0.35 & 0.20 & 0.43 & 0.14 & 0.26 & 0.26 & 0.37 & 0.13 & - & 0.46 & 0.29 & 0.34 \\
\hline 15 & 3.09 & 0.56 & 0.28 & 0.38 & 0.28 & 0.30 & 0.20 & 0.22 & 0.23 & 0.31 & 0.38 & 0.56 & 0.35 & 0.16 & 0.43 & - & 0.36 & 0.33 \\
\hline 16 & 2.76 & 0.51 & 0.19 & 0.22 & 0.47 & 0.38 & 0.19 & 0.31 & 0.28 & 0.55 & 0.22 & 0.17 & 0.22 & 0.53 & 0.34 & 0.43 & - & 0.29 \\
\hline 17 & 2.69 & 0.46 & 0.28 & 0.14 & 0.24 & 0.10 & 0.20 & 0.40 & 0.36 & 0.28 & 0.14 & 0.23 & 0.27 & 0.17 & 0.37 & 0.38 & 0.37 & - \\
\hline
\end{tabular}

物种编号含义见表1。Species codes are the same as in Table 1 . 


\section{3 讨论}

\section{1 金钟藤群落优势种种间联结关系}

金钟藤群落中 17 个优势种间的关联性较弱, 也 进一步说明群落仍处于动态的演替阶段, 尚未达到 稳定状态。136个种对中, 有69个表现出正相关性, 67 个表现出负相关性或处于独立状态(表2), 说明在 当前演替阶段, 虽然金钟藤群落中大部分物种对资 源的利用情况基本相似，但也存在部分物种对水、 光照、土壤养分等资源竞争的情况, 并且这种竞争 将随着金钟藤入侵的加剧和资源的耗竭会愈发明 显(刘海等, 2017)。其中, 黄椿木姜子-九节、岭南山 竹子-显脉杜英、倒吊笔-黄椿木姜子、岭南山竹子 -银柴、鸭脚木一银柴表现出显著的正关联性(表2, 图1), 说明这些物种的生态习性较为相似, 能够相 互促进、共同生长(王伯荪和彭少麟, 1985; 周先叶 等, 2000; 李建民等, 2001)。倒吊笔一肉实树、翻白 叶树-岭南山竹子、白楸-九节、翻白叶树-鸭脚木、 假柿木姜子-肉实树和九节-楝叶吴荣英则表现为 显著的负关联(表2, 图1), 可能是由于生态习性存 在较大的差异(Greig-Smith, 1983; 房飞等, 2012), 对群落微环境的需求不一致; 也可能是由于金钟藤 的入侵，使得环境营养空间和资源大大减少，从而 造成这几个物种为争夺资源产生竞争(Roxburgh \& Chesson, 1998; 郭圭金等, 2011; 赵彩莉等, 2013)。

作为入侵物种, 金钟藤不可避免地与群落中的 其他物种在资源利用方面存在竞争, 其根系粗壮发 达且地上部分繁茂, 能大肆抢夺养分, 并可通过分 泌化感物质来影响其他物种的生长(吴林芳等, 2007; Huang et al, 2013)。金钟藤与芳槁润楠、黄椿木姜 子、岭南山竹子、显脉杜英、鸭脚木和银柴存在非 常紧密的关联性和较高的相伴出现机率(图2), 一方 面说明这几个物种与金钟藤具有相同或相似的资 源利用方式, 以及对环境的变化有相似的适应性 (Greig-Smith, 1983; 王伯荪和彭少麟, 1985; 李建民 等, 2001); 另一方面也说明这些物种对金钟藤的抵 抗能力较强, 尽管金钟藤对群落具有极大的危害性, 但这些物种仍能不被排斥出群落(刘海等, 2017)。

\section{2 金钟藤群落稳定性}

金钟藤所在群落中主要优势物种间总体上呈 不显著的正关联关系 $(V R=1.30, W=62.36)$ 。群落稳 定比值远离(20,80), 群落的相对稳定性较差(图3)。
说明金钟藤入侵群落中, 各物种间尚未形成稳定协 调的搭配和紧密的耦合关系; 群落的结构和功能还 不稳定, 物种组成和数量结构也还处于动态调节中, 离顶极群落有一定距离(康冰等, 2006; 黄祥童等, 2015)。这意味着金钟藤的入侵会加剧群落的不稳定 性, 这一结论在其他入侵种的研究中得到了证实, 如空心莲子草(Alternanthera philoxeroides) (郭连金 和王涛, 2009)、苏门白酒草(Conyza sumatrensis) (郭 连金, 2011)、紫茎泽兰(Eupatorium adenophorum)等 (刘海等, 2017)的入侵均会进一步导致群落稳定性 的明显下降。

种间竞争、环境压力和人类干扰等因素均会对 植物群落稳定性产生影响(刘珏宏等, 2010; 房飞等, 2012; 潘高等, 2017; 叶权平等, 2018)。金钟藤多分 布于容易受干扰或已受到一定干扰的地方，如旅游 景区、废弃的停车场和庭园周围, 说明在金钟藤入 侵前，土著植物群落由于长期受到干扰而打破了原 有的平衡与稳定, 给金钟藤的入侵创造了机会。在 金钟藤入侵后，由于其较强的生态适应性，可在短 时间内大面积繁殖覆盖群落, 其分泌的化感物质也 具有抑制作用，进一步加剧了群落的不稳定性(吴 林芳等, 2007; 王伯荪等, 2009; Huang et al, 2013; 李晓霞等, 2014)。因此, 保持群落的稳定有利于减 少金钟藤的入侵机会。

\section{3 金钟藤群落优势种生态位}

在金钟藤典型分布群落中, 金钟藤占据最大的 生态位宽度，是群落的绝对优势种(表3)。说明金钟 藤对环境资源的利用更为充分, 对环境的适应能力 也更强，从而拥有较强的竞争力(康冰等, 2006; 刘 巍和曹伟, 2011), 加上其较强的繁殖能力, 是其成 功入侵并进一步蔓延的主要原因。芳槁润楠、黄椿 木姜子、显脉杜英和银柴也占据了较大的生态位宽 度(表3), 说明这些物种在群落的演替过程中也能占 据较大的优势, 并稳定存在于演替的各个阶段(刘 巍和曹伟, 2011; 辛小娟等, 2011)。其他物种的生态 位宽度较小(表3), 说明这些物种具有一定的特化程 度, 在整个演替过程中, 其环境适应性、资源利用能 力和竞争力相对较弱(刘巍和曹伟, 2011; 辛小娟等, 2011), 随着群落的演替发展可能会被逐渐排除出。

多数关于生态位的研究均表明, 生态位宽度较 大的物种常常伴随较高的生态位重叠，而生态位窄 的物种间的生态位重叠则比较低(Walker, 1995; 柴 
宗政等, 2012; 铁军等, 2015)。本研究进一步证实了 这个观点。在群落中, 金钟藤的生态位宽度最大, 与伴生物种的生态位重叠也较高。生态位宽度较大 的伴生物种间, 比如黄椿木姜子与九节, 芳槁润楠 与鸭脚木等也存在较高的生态位重叠。这些物种对 资源的综合利用能力较强, 对环境因子的变化具有 较好的生态适应性, 分布范围较广, 与其他物种产 生资源竞争的机会也就较多, 因而具有较高的生态 位重叠(张继义等, 2003; 刘巍和曹伟, 2011; 李燕芬 等, 2014)。但生态位宽度与生态位重叠的关系非绝 对的正相关, 受空间异质性的影响, 或者是生长需 求存在差异或互补, 生态位宽度大的物种如芳槁润 楠、黄椿木姜子、显脉杜英和银柴等存在较低的生 态位重叠, 而生态位宽度较小的物种如假柿木姜 子、楝叶吴荣英、海南菜豆树 (Radermachera hainanensis)和翻白叶树等出现较高的生态位重叠(史作 民等, 1999; 辛小娟等, 2011; 吴会峰等, 2017)。

在群落中, 伴生物种间的生态位重叠较低, 说 明竞争较小或不存在竞争关系; 金钟藤与伴生物种 间则存在较严重的生态位重叠, 这与金钟藤的入侵 性分不开。以往的研究(洪思思等, 2008; 陈彤等, 2012)表明, 外来植物的入侵会导致本土植物间的 生态位重叠明显降低, 同时大大地增强了本土植物 与入侵植物之间的竞争排除作用, 本研究进一步证 实了这个观点。根据种间联结和生态位的分析结果, 金钟藤的伴生物种可分为四大生态组: 第一组包括 芳槁润楠、黄椿木姜子、显脉杜英、鸭脚木和银柴 5 个物种, 这些物种与金钟藤存在较强的正联结关 系和较高的生态位重叠, 对金钟藤的入侵具有一定 的抵抗作用, 且这几个物种彼此间并不存在竞争关 系。第二组为岭南山竹子, 该物种与金钟藤呈正联 结关系但生态位重叠较低, 说明两者的空间生态位 可能出现了分化并形成互补关系。金钟藤入侵后通 常会极大地增加群落的郁闭度, 而岭南山竹子能生 长于郁闭度较高的密林、沟谷间, 因此其生长不受 影响。第三组包括白楸、倒吊笔、假苹婆、假柿木 姜子、楝叶吴茱英和水锦树, 这些物种与金钟藤呈 负联结且生态位重叠指数较低, 说明与金钟藤的生 态习性差异性较大而不能一起生长。第四组包括翻 白叶树、海南菜豆树、九节和肉实树 4 个物种, 这些 物种与金钟藤呈负联结但生态位重叠较高, 说明与 金钟藤对资源的利用需求存在激烈的竞争关系。

\section{4 防治建议}

目前对金钟藤的防治主要有物理防治和化学 防治等, 物理防治需要耗费大量的人力物力, 而化 学控制容易造成污染(童国建等, 2005; 吴林芳等, 2007)。本研究通过对金钟藤与群落中其他优势种间 的联结关系以及生态位的分析，对金钟藤的防治有 两点建议: (1)加强对林区群落的保护，减少外来干 扰。以往的研究表明, 干扰会严重破坏群落中各物 种的组成及种间原有的关系，使物种产生强烈的竞 争, 不但导致群落物种多样性和稳定性发生变化, 甚至会导致外来物种的入侵(刘艳红和赵慧勋, 2000; 郝建锋等, 2016)。因此，加强对林区的保护，减少干 扰因素, 既是对原有群落物种的保护, 也是预防外 来物种入侵的最好措施。(2)栽种与金钟藤存在竞争 关系的树种，用于金钟藤的生态控制以及植被恢 复。对于已被金钟藤入侵的群落, 建议大量栽种第 一生态组的物种，该生态组的物种既对金钟藤的入 侵具有抵抗作用，在资源不足时又能与其形成激烈 的竞争，遏制金钟藤的蔓延，能达到较好的生态控 制效果; 对于已进行金钟藤砍除的群落, 建议大量 栽种第四生态组的物种来进行群落的植被恢复。

致谢: 感谢李超、陈庆、李时兴在样地调查过程中 给予的热情帮助; 感谢张金龙博士在数据分析过程 中的热情帮助。

\section{参考文献}

Callaway RM, Maron J (2006) What have exotic plant invasions taught us over the past 20 years. Trends in Ecology and Evolution, 21, 369-374.

Cárdenas RE, Valencia R, Kraft NJB, Argoti A, Dangles O, Swenson N (2014) Plant traits predict inter- and intraspecific variation in susceptibility to herbivory in a hyperdiverse neotropical rain forest tree community. Journal of Ecology, 102, 939-952.

Chai ZZ, Wang DX, Zhang LN, Zhang Y, Huang QP, Wu H (2012) Niche characteristics of main plant populations in natural Pinus tabulaeformis communities in Qinling Mountains, Northwest China. Chinese Journal of Ecology, 31, 1917-1923. (in Chinese with English abstract) [柴宗政, 王 得祥, 张丽楠, 张洋, 黄青平, 吴吴 (2012) 秦岭山地天 然油松群落主要植物种群生态位特征. 生态学杂志, 31, 1917-1923.]

Chen YK, Yang XB, Li DH, Nong SQ, Lü XB, Lü JJ, Yang M, Li XC (2011) Interspecific associations among dominant plant populations in Keteleeria hainanensis communities in 
Bawangling, Hainan Island. Plant Science Journal, 29, 278-287. (in Chinese with English abstract) [陈玉凯, 杨小 波, 李东海, 农寿千, 吕晓波, 吕洁杰, 杨民, 李小成 (2011) 海南霸王岭海南油杉群落优势种群的种间联结性 研究. 植物科学学报, 29, 278-287.]

Chen T, Liu WL, Zhang CB, Wang J (2012) Effects of Solidago canadensis invasion on dynamics of native plant communities and their mechanisms. Chinese Journal of Plant Ecology, 36, 253-261. (in Chinese with English abstract) [陈彤, 刘文莉, 张崇邦, 王江 (2012) 加拿大一枝黄花入 侵对本土植物群落动态的影响及其机制. 植物生态学报, 36, 253-261. ]

Fang F, Hu YK, Zhang W, Gong YM, Liu YY, Yang XJ (2012) Numerical analysis of inter-specific relationships in alpine steppe community in Bayanbulak. Acta Ecologica Sinica, 32, 1898-1906. (in Chinese with English abstract) [房飞, 胡玉昆, 张伟, 公延明, 柳妍妍, 杨秀娟 (2012) 高寒草 原植物群落种间关系的数量分析. 生态学报, 32, 1898-1906.]

Finch D (2012) Climate Change in Grasslands, Shrublands and Deserts of the Interior American West: A Review and Needs Assessment. USDA Forest Service Rocky Mountain Research Station, Fort Collins.

Gao RM, Shi XD, Guo YD (2012) Community stability evaluation of riparian forest of the upper reaches of Wenyuhe in Shanxi, China. Chinese Journal of Plant Ecology, 36, 491503. (in Chinese with English abstract) [高润梅, 石晓东, 郭跃东 (2012) 山西文峪河上游河岸林群落稳定性评价. 植物生态学报, 36, 491-503.]

Gleason HA (1926) The individualistic concept of the plant association. Bulletin of the Torrey Botanical Club, 53, 7-26.

Godron M (1972) Some aspects of heterogeneity in grasslands of Cantal. Statistical Ecology, 3, 397-415.

Greig-Smith P (1983) Quantitative Plant Ecology, 3rd edn. Blackwell Scientific Publications, Oxford.

Guo LJ (2011) Impacts to the interspecies association and stability of native community invaded by an exotic plant Conyza sumatrensis. Subtropical Plant Science, 40(2), 18-23. (in Chinese with English abstract) [郭连金 (2011) 苏门白酒草对乡土植物群落种间联结性及稳定性的影响. 亚热带植物科学, 40(2), 18-23.]

Guo LJ, Wang $T$ (2009) Impact of invasion of exotic plant Alternanthera philoxeroides on interspecies association and stability of native plant community. Chinese Journal of Eco-Agriculture, 17, 851-856. (in Chinese with English abstract) [郭连金, 王涛 (2009) 空心莲子草入侵对乡土 植物群落种间联结性及稳定性的影响. 中国生态农业学 报, 17, 851-856.]

Guo YX, Hu YN, Kong LT, Wang DX, Yang GH (2011) Interspecific association and correlation of main plant species in subalpine meadow of Zibai Mountain. Chinese Journal of Ecology, 30, 1775-1780. (in Chinese with English abstract) [ 郭圭金金, 胡有宁, 孔令童, 王得祥, 杨改河 (2011) 紫柏山亚高山草甸群落的种间关联和相关分析. 生态学杂志, 30, 1775-1780.]
Hao JF, Li Y, Qi JQ, Pei ZL, Huang YJ, Jiang Q, Chen Y (2016) Effects of anthropogenic disturbances on the species diversity and niche of the dominant populations in a Castanopsis fargesii secondary forest community in Bifengxia, Sichuan. Acta Ecologica Sinica, 36, 7678-7688. (in Chinese with English abstract) [郝建锋, 李艳, 齐锦秋, 裴曾莉, 黄 雨佳, 蒋倩, 陈亚 (2016) 人为干扰对碧峰峡栲树次生林 群落物种多样性及其优势种群生态位的影响. 生态学报, 36, 7678-7688.]

Hong SS, Miao CC, Fang BJ, Hu RY, Ding BY (2008) On species diversity, niche breath and interspecies association in communities invaded by Spermacoce latifolia Zhejiang Province. Journal of Wuhan Botanical Research, 26, 501508. (in Chinese with English abstract) [洪思思, 缪崇崇, 方本基, 胡仁勇, 丁炳扬 (2008) 浙江省阔叶丰花草入侵 群落物种多样性、生态位及种间联结研究. 武汉植物学研 究, 26, 501-508.]

Hu XC, Gao HH (2008) General situation and protection of tropical natural forests in Hainan Island. Modern Agriculture Science and Technology, (22), 76-77. (in Chinese) [胡小婵, 高宏华 (2008) 海南岛热带天然林概况及其保护. 现代 农业科技, (22), 76-77.]

Huang QQ, Shen YD, Li XX, Fan ZW, Li MG, Cheng HT (2013) Native expanding Merremia boisiana is not more allelopathic than its non-expanding congener $M$. vitifolia in the expanded range in Hainan. American Journal of Plant Sciences, 4, 774-779.

Huang QQ, Shen YD, Fan ZW, Li XX, Song X, Cheng HT, Hou YP (2013) Effects of soil from different forest types in Wuzhi Mountain on the seedling growth of Merremia boisiana. Ecology and Environment Sciences, 22, 95-99. (in Chinese with English abstract) [黄乔乔, 沈奕德, 范志伟, 李晓霞, 宋釒鍂, 程汉亭, 侯玉平 (2013) 五指山不同林型 土壤对金钟藤幼苗生长的影响. 生态环境学报, 22, 95-99.]

Huang XT, Wang SX, Huang BJ, Yin H, Cui KF, Zhao W, Fan YG, Gu DF (2015) Analyses of community stability and inter-specific associations between the rare plant Phyllitis scolopendrium and its associated species. Acta Ecologica Sinica, 35, 80-90. (in Chinese with English abstract) [黄祥 童, 王绍先, 黄炳军, 尹航, 崔凯峰, 赵伟, 范宇光, 顾德 峰 (2015) 珍稀植物对开硕与其伴生物种的联结性及群 落稳定性. 生态学报, 35, 80-90.]

Hubalek Z (1982) Coeffident of association and similarity based on binary data: An evaluation. Biological Reviews, 57, 669-689.

Hutchinson GE (1957) Concluding remarks: Cold spring harbor symposium of quantitative biology. Quantitative Biology, 22, 415-427.

Kang B, Liu SR, Zhang GJ, Chang JG, Wen YG, Ma JM, Hao WF (2006) Carbon accumulation and distribution in Pinus massoniana and Cunninghamia lanceolata mixed forest ecosystem in Daqingshan, Guangxi of China. Acta Ecologica Sinica, 26, 1320-1329. (in Chinese with English abstract) [康冰, 刘世荣, 张广军, 常建国, 温远光, 马姜明, 郝文 
芳 (2006) 广西大青山南亚热带马尾松、杉木混交林生态 系统碳素积累和分配特征. 生态学报, 26, 1320-1329.]

Levins R (1968) Evolution in Changing Environments: Some Theoretical Explorations. Princeton University Press, Princeton.

Li JM, Xie F, Chen CJ, Zhang SY, Xiao RH, Zhao DZ (2001) Interspecific association of dominant species in Betula luminifera natural forest communities of Shaowu, Fujian Province. Chinese Journal of Applied Ecology, 12, 168-170. (in Chinese with English abstract) [李建民, 谢芳, 陈存及, 张思玉, 肖日红, 赵大洲 (2001) 光皮桦天然林群落优势 种群的种间联结性研究. 应用生态学报, 12, 168-170.]

Li L, Xu ZF, Wei X, Cao HL, Su J, Ye WH (2008) Physiological compare Merremia boisiana with Pueraria lobata under drought stress and rewatering conditions. Guihaia, 28, 806-810. (in Chinese with English abstract) [李玲, 徐志防, 韦霄, 曹洪麟, 粟娟, 叶万辉 (2008) 金钟藤和葛藤在干 旱与复水条件下的生理比较. 广西植物, 28, 806-810.]

Li SF, Liu WD, Su JR, Zhang ZJ (2011) Niches and interspecific associations of dominant tree populations at different restoration stages of monsoonal broad-leaved evergreen forest. Chinese Journal of Ecology, 30, 508-515. (in Chinese with English abstract) [李帅锋, 刘万德, 苏建荣, 张 志钧 (2011) 季风常绿阔叶林不同恢复阶段乔木优势种 群生态位和种间联结. 生态学杂志, 30, 508-515.]

Li XX, Huang QQ, Fan ZW, Shen YD, Cheng HT, Liu LZ (2014) Chemical compositions and allelopathic potential of volatile oil from Merremia boisiana. Chinese Journal of Tropical Crops, 35, 1643-1647. (in Chinese with English abstract) [李晓霞, 黄乔乔, 范志伟, 沈奕德, 程汉亭, 刘 丽珍 (2014) 金钟藤叶挥发油化学成分分析及其化感潜 力研究. 热带作物学报, 35, 1643-1647.]

Li YF, Tie J, Zhang GP, Guo H (2014) Niche characteristics of an artificial Pinus tabuliformis forest in Manghe National Nature Reserve of Shanxi. Chinese Journal of Ecology, 33, 2905-2912. (in Chinese with English abstract) [李燕芬, 铁 军, 张桂萍, 郭华 (2014) 山西蟒河国家级自然保护区人 工油松林生态位特征. 生态学杂志, 33, 2905-2912.]

Liang WS, Fang TS, Yu HB, Wang Z (2012) Risk analysis of Merremia boisiana in China. Guangdong Forestry Science and Technology, 26(3), 42-45. (in Chinese with English abstract) [梁玮莎, 方天松, 余海滨, 王忠 (2012) 金钟藤 在中国的风险性分析. 广东林业科技, 26(3), 42-45.]

Liu H, Du RW, Wang Y, Chen YL, Wu YK, Yuan L (2017) Effects of Eupatorium adenophorum on interspecific association and the stability of companion species in Liangshan Prefecture of Sichuan Province. Acta Ecologica Sinica, 37, 5031-5038. (in Chinese with English abstract) [刘海, 杜如 万, 王勇, 陈玉蓝, 吴叶宽, 袁玲 (2017) 紫茎泽兰对四 川省凉山州共生植物种间联结性及稳定性的影响. 生态 学报, 37, 5031-5038.]

Liu W, Cao W (2011) Niche characteristics of main plant species in spruce-fir forests in Changbai Mountains. Chinese Journal of Ecology, 30, 1766-1774. (in Chinese with English abstract) [刘巍, 曹伟 (2011) 长白山云冷杉群落
主要种群生态位特征. 生态学杂志, 30, 1766-1774.]

Liu YH, Zhao HX (2000) Advances in theory of disturbance and species diversity preservation. Journal of Beijing Normal University, 22(4), 101-105. (in Chinese with English abstract) [刘艳红, 赵惠勋 (2000) 干扰与物种多样性维 持理论研究进展. 北京林业大学学报, 22(4), 101-105.]

Liu YH, Gao H, Zhang LH, Chen LP, Zhao NX, Gao YB (2010) Comparative analysis of inter-specific association within the Stipa grandis-S. krylovii community in typical steppe of Inner Mongolia, China. Chinese Journal of Plant Ecology, 34, 1016-1024. (in Chinese with English abstract) [刘珏宏, 高慧, 张丽红, 陈丽萍, 赵念席, 高玉葆 (2010) 内蒙古锡林郭勒草原大针茅-克氏针茅群落的种间关联 特征分析. 植物生态学报, 34, 1016-1024.]

Pan G, Zhang HP, Pan D (2017) Interspecific associations of dominant plant populations in a mid-subtropical Choerospondias axillaris forest. Chinese Journal of Ecology, 36, 892-901. (in Chinese with English abstract) [潘高, 张合平, 潘登 (2017) 中亚热带南酸苯林优势种群的种间联结性. 生态学杂志, 36, 892-901.]

Peng SL, Zhou HC, Guo SC, Huang ZL (1999) Studies on the changes in interspecific association of zonal vegetation in Dinghushan. Acta Botanica Sinica, 41, 1239-1244. (in Chinese with English abstract) [彭少麟, 周厚诚, 郭少聪, 黄忠良 (1999) 鼎湖山地带性植被种间联结变化研究. 植物学报, 41, 1239-1244.]

Pianka ER (1973) The structure of lizzard communities. Annual Review of Ecology and Systematics, 89, 1561-1565.

Pimentel D, Lach L, Zuniga R, Morrison D (2000) Environmental and economic costs of nonindigenous species in the United States. BioScience, 50, 53-65.

Rosenthal G (2003) Selecting target species to evaluate the success of wet grassland restoration. Agriculture, Ecosystems and Environment, 98, 227-246.

Roxburgh SH, Chesson P (1998) A new method for detecting species associations with spatially autocorrelated data. Ecology, 79, 2180-2192.

Schluter D (1984) A variance test for detecting species associations, with some example applications. Ecology, 65, 9981005.

Schoener TW (1974) Resource partitioning in ecological communities. Science, 185, 27-39.

Shi ZM, Cheng RM, Liu SR (1999) Niche characteristics of plant populations in deciduous broad-leaved forest in Baotianman. Chinese Journal of Applied Ecology, 10, 265-269. (in Chinese with English abstract) [史作民, 程瑞梅, 刘世 荣 (1999) 宝天曼落叶阔叶林种群生态位特征. 应用生 态学报, 10, 265-269.]

Tang JM, Ai XR, Yi YM, Li L, Xu HM, Song YL (2012) Niche dynamics during restoration process for the dominant tree species in montane mixed evergreen and deciduous broadleaved forests at Mulinzi of southwest Hubei. Acta Ecologica Sinica, 32, 6334-6342. (in Chinese with English abstract) [汤景明, 艾训儒, 易咏梅, 李玲, 徐红梅, 宋亚 丽 (2012) 鄂西南木林子常绿落叶阔叶混交林恢复过程 
中优势树种生态位动态. 生态学报, 32, 6334-6342.]

Thevathasan NV, Gordon AM (2004) Ecology of tree intercropping systems in the north temperate region: Experiences from southern Ontario, Canada. Agroforestry Systems, 61, 257-268.

Tie J, Li YF, Wang X, Bai FL, Cui FT, Jin S (2015) Species diversity of forest community in Macaca mulatta habitat in Lishan National Nature Reserve, Shanxi. Chinese Journal of Ecology, 34, 3009-3015. (in Chinese with English abstract) [铁军, 李燕芬, 王霞, 白凤麟, 崔方天, 金山 (2015) 山 西历山国家级自然保护区狝猴栖息地森林群落物种多样 性. 生态学杂志, 34, 3009-3015.]

Tong GJ, Chen MR, Yu HB (2005) Preliminary report on the damage of Merremia boisiana. Forest Pest and Disease, 24(3), 17-18. (in Chinese with English abstract) [童国建, 陈沐荣, 余海滨 (2005) 金钟藤的危害性初报. 中国森林 病虫, 24(3), 17-18.]

Walker BH (1995) Conserving biological diversity through ecosystem resilience. Conservation Biology, 9, 747-752.

Wang BS, Li MG, Liao WB (2005) Geographical distribution of Merremia boisiana. Ecology and Environment, 14, 451-454. (in Chinese with English abstract) [王伯䔉, 李鸣 光, 廖文波 (2005) 金钟藤的地理分布. 生态环境, 14 , 451-454.]

Wang BS, Peng SL (1985) Studies on the measuring techniques of interspecific association of lower-subtropical evergreen-broadleaved forests. I. The exploration and the revision on the measuring formulas of interspecific association. Acta Phytoecologica et Geobotanica Sinica, 9, 32-43. (in Chinese with English abstract) [王伯䔉, 彭少麟 (1985) 南亚热带 常绿阔叶林种间联结测定技术研究. I. 种间联结测式的 探讨与修正. 植物生态学与地植物学从刊, 9, 32-43.]

Wang BS, Peng SL, Li DJ, Zhou T (2009) Research progress on Merremia boisiana. Chinese Journal of Ecology, 28, 2360-2365. (in Chinese with English abstract) [王伯䔉, 彭 少麟, 李代江, 周婷 (2009) 金钟藤研究述评. 生态学杂 志, 28, 2360-2365.]

Wang BS, Qiu HX, Liao WB, Li MG, Ding MY, Peng SL (2007) Revision and additional notes on Merremia boisiana and M. boisiana var. fulvopilosa (Convolvulaceae). Guihaia, 27, 527-536. (in Chinese with English abstract) [王伯䔉, 丘华兴, 廖文波, 李鸣光, 丁明艳, 彭少麟 (2007) 金钟 藤分类考证及补充描述. 广西植物, 27, 527-536.]

Wu HF, Song LJ, Du F, Hao WF (2017) Niche of main populations and environmental interpretation in grassland of Loess Plateau hilly region, China. Chinese Journal of Applied Ecology, 28, 3494-3504. (in Chinese with English abstract) [吴会峰, 宋丽娟, 杜峰, 郝文芳 (2017) 黄土丘 陵区草地主要种群生态位及其环境解释. 应用生态学报, 28, 3494-3504.]

Wu LF, Liang YQ, Chen K, Li ZC, Cao HL (2007) Damage and prevention of Merremia boisiana in Hainan Province,
China. Guangdong Forestry Science and Technology, 23(1), 83-86. (in Chinese with English abstract) [吴林芳, 梁永勤, 陈康, 李忠超, 曹洪麟 (2007) 金钟藤在海南的危害与防 治. 广东林业科技, 23(1), 83-86.]

Xin XJ, Yang YB, Wang G, Ren ZW, Chu CJ, Zhang RY (2011) Niche dynamics of plant community succession and grassland quality index on zokor mounds. Chinese Journal of Ecology, 30, 700-706. (in Chinese with English abstract) [辛小娟, 杨䒯博, 王刚, 任正炜, 储诚进, 张仁懿 (2011) 鼠鼠土丘植物群落演替生态位动态及草地质量指数. 生 态学杂志, 30, 700-706.]

Xu MH, Liu M, Zhai DT, Liu T (2016) A review of contents and methods used to analyze various aspects of plant interspecific associations. Acta Ecologica Sinica, 36, 8224-8233. (in Chinese with English abstract) [徐满厚, 刘 敏, 翟大誈, 刘粀 (2016) 植物种间联结研究内容与方法 评述. 生态学报, 36, 8224-8233.]

Ye QP, Zhang WH, Yu SC, Xue WY (2018) Interspecific association of the main tree populations of the Quercus acutissima community in the Qiaoshan forest area. Acta Ecologica Sinica, 38, 3165-3174. (in Chinese with English abstract) [叶权平, 张文辉, 于世川, 薛文艳 (2018) 桥山 林区麻栎群落主要乔木种群的种间联结性. 生态学报, 38, 3165-3174.]

Zhang JY, Zhao HL, Zhang TH, Zhao XY (2003) Niche dynamics of main populations of plants communities in the restoring succession process in Horqin Sandy Land. Acta Ecologica Sinica, 23, 2741-2746. (in Chinese with English abstract) [张继义, 赵哈林, 张铜会, 赵学勇 (2003) 科尔 沁沙地植物群落恢复演替系列种群生态位动态特征. 生 态学报, 23, 2741-2746.]

Zhao CL, Zhang F, Pang CH, Wang HM, Fan X (2013) Interspecific association of dominant species of Amaranthus retroflexus L. community. Bulletin of Botanical Research, 33, 454-460. (in Chinese with English abstract) [赵彩莉, 张峰, 庞春花, 王慧敏, 范晓 (2013) 反枝苋群落优势种 的种间关联性分析. 植物研究, 33, 454-460.]

Zheng YR (2000) Comparison of methods for studying stability of forest community. Scientia Silvae Sinicae, 36(5), 28-32. (in Chinese with English abstract) [郑元润 (2000) 森林群 落稳定性研究方法初探. 林业科学, 36(5), 28-32.]

Zhou XY, Wang BS, Li MG, Zan QJ (2000) An analysis of interspecific associations in secondary succession forest communities in Heishiding Natural Reserve, Guangdong Province. Acta Phytoecologica Sinica, 24, 332-339. (in Chinese with English abstract) [周先叶, 王伯䔉, 李鸣光, 处启杰 (2000) 广东黑石顶自然保护区森林次生演替过 程中群落的种间联结性分析. 植物生态学报, 24, 332-339.]

\section{(责任编委：蔵润国 责任编辑：黄祥忠)}

